\section{DGAKI - neue Abkürzung und neues Logo}

$\mathrm{D}$ ie Deutsche Gesellschaft für Allergologie und klinische Immunologie hat sich zum 1. Juli 2005 eine neue Abkürzung gegeben - aus DGAI wurde DGAKI. Welche Gründe für den Wechsel gab es, nachdem die alte $\mathrm{Ab}$ kürzung DGAI über 30 Jahre Bestand hatte?

Am 17. Oktober 1963 war der Beschluss zur Namensänderung von „Gesellschaft für Allergologie“, gegründet am 17. Juni 1951 in Frankfurt am Main, in „Deutsche Gesellschaft für Allergologie und Immunitätsforschung" auf der neunten Jahrestagung in Bad Lippspringe gefasst worden. Als Abkürzung für den neuen Namen wurde damals DGAI festgelegt. Dieses Kürzel konnte auch nach erneuter Umbenennung in „Deutsche Gesellschaft für Allergologie und klinische Immunologie“ im Jahr 1996 beibehalten werden.
Die im April 1953 gegründete „Deutsche Gesellschaft für Anästhesiologie und Intensivmedizin e. V. (DGAI)" machte nunmehr die Deutsche Gesellschaft für Allergologie und klinische Immunologie darauf aufmerksam, dass DGAI die geschützte Bezeichnung ihrer Fachgesellschaft ist. So war die Deutsche Gesellschaft für Allergologie und klinische Immunologie wegen der älteren Rechte der Anästhesiologen zur Änderung gezwungen, das Ergebnis lautet DGAKI. Wir hoffen, dass die Mitglieder der DGAKI sich rasch an die neue Bezeichnung gewöhnen werden und dass das Interesse auch der Nichtmitglieder an der DGAKI weiter wachsen möge.

Das Kürzel DGAKI ist übrigens nicht ganz neu, unter www.dgaki.de war schon immer die Homepage der Gesellschaft zu finden.

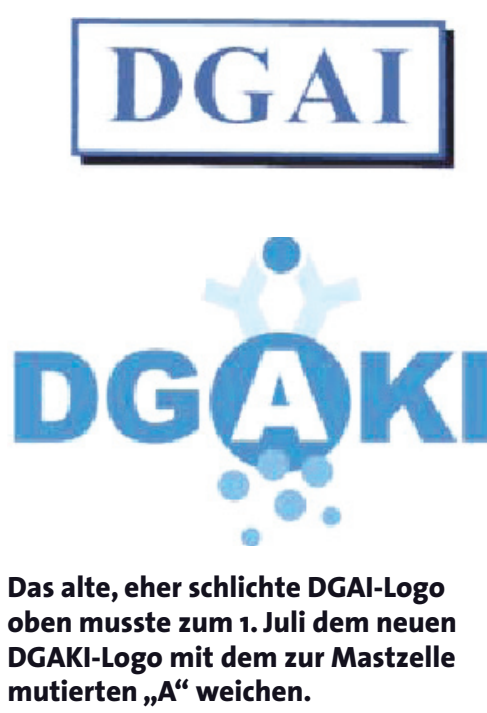

Mit der neuen Ankürzung verbunden war die Wahl eines neuen Logos. Die DGAKI hofft auf positive Resonanz bei ihren Mitgliedern.

Prof. Dr. Gerhard Schultze-Werninghaus, Bochum, Präsident der DGAKI

\section{Ad multos annos: Professor Dr. Gert Kunkel}

A m 23. September 2005 vollendet Prof. Dr. med. Gert Kunkel sein 70. Lebensjahr. Die Deutsche Gesellschaft für Allergologie und klinische Immunologie gratuliert ihrem herausragenden Mitglied zu diesem runden Geburtstag.

Prof. Kunkel ist wie wenige weit über seinen offiziellen Ruhestand hinaus beruflich und wissenschaftlich Vorbild für uns alle - die letzte internationale Publikation stammt vom Januar 2005 und ist seinem Forschungsthema der letzten Jahre gewidmet, den Neurotrophinen (Rost B et al. Regul Pept 2005; 124: 10-25). Damit geht der Blick zurück über ein jahrzehntelanges Interesse an der Regulation des Atemwegstonus, erstmals publiziert vor 30 Jahren (Kunkel G et al. Allergol Immunopathol 1975; 6: 445-56).

Prof. Kunkel war von 1971 bis 2002 Leiter der Abteilung für klinische Immunologie und Asthma-Poliklinik des Klinikums Rudolf Virchow, Freie Universi- tät Berlin, seit 1994 Virchow-Klinikum der Charité, Humboldt-Universität Berlin, als einer der herausragenden internistisch-pneumologisch orientierten Allergologen unseres Landes. Seine umfassenden wissenschaftlichen Interessen, in Verbindung mit dem Wunsch, aus Ber-

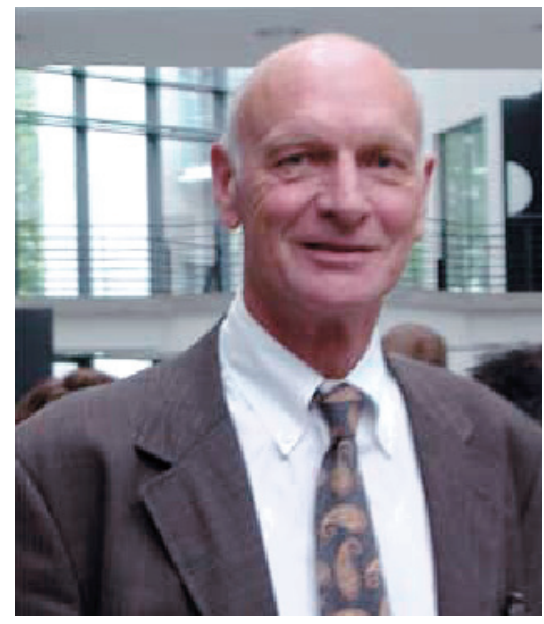

Prof. Dr. med. Gert Kunkel lin heraus auch internationale Verbindungen zu schaffen, wurden anerkannt durch Ehrungen, wie die Ernennung zum Honorarprofessor für Innere Medizin an der Universität von Südflorida, Tampa, USA, 1984, und zum Ehrenprofessor der Medizinischen Fakultät der Universität Skopje, Mazedonien, 1998. Der Dr.-Karl-Heyer-Preis wurde Prof. Kunkel 1992 verliehen.

Höhepunkt der Anerkennung seines internationalen wissenschaftlichen Engagements war die Verleihung der Kongresspräsidentschaft der Europäischen Akademie für Allergologie und klinische Immunologie, Berlin 1989.

Prof. Kunkel ist der DGAKI während seines gesamten beruflichen Lebens eng verbunden gewesen. Er war Präsident der DGAKI von 1990-1993, bis 1996 Vizepräsident, und ist seit 1996 Ehrenmitglied. Er erhielt 2004 die höchste Ehrung der DGAKI, die Karl-Hansen-Medaille. Die DGAKI wünscht Prof. Kunkel viele weitere beruflich und privat erfreuliche Jahre.

Prof. Dr. Gerhard Schultze-Werninghaus, 